\title{
Life of the dust macroparticles in storage rings
}

\author{
S. Heifets, Qing Qin, ${ }^{*}$ and M. Zolotorev ${ }^{\dagger}$ \\ Stanford Linear Accelerator Center, Stanford University, Stanford, California 94309, USA
}

(Received 14 April 2005; published 10 June 2005)

\begin{abstract}
The sudden drop of the beam lifetime and bursts of the background radiation were detected in many machines and associated with microscopic dust particles. We present the model of the dust particle dynamics explaining the long time of the dust events observed in the PEP-II B-factory and BEPC-II machines.
\end{abstract}

DOI: 10.1103/PhysRevSTAB.8.061002

PACS numbers: 29.27.Bd

\section{INTRODUCTION}

The sudden drop of the beam lifetime and bursts of the background radiation were detected in many machines and associated with microscopic dust particles. The dust events could last several tens of second. Dust events were observed in many machines including such different machines as PEP-II B-factory and Beijing Electron-Positron Collider (BEPC) where the revolution frequency and the beam current are different by an order of magnitude. Some relevant parameters of the machines and notations are given in the Table I. In PEP-II, the lifetime in the dust events was reduced by approximately $10 \%$. BEPC could operate in dual mode as a collider and the synchrotron radiation (SR) light source. In the first case, both $e^{-}$and $e^{+}$ beams are stored in the same beam pipe using electrostatic separators. In the single-beam operations, reduction of the beam life was observed from the normal lifetime $\tau \simeq$ $8-10 \mathrm{~h}$ to $2 \mathrm{~h}$ or even to few minutes. That happened randomly, at wide range of beam current, both in singlebunch and multibunch cases, were not reproducible with the same machine parameters, and never happened with the positron beam. The lifetime can recover by itself or, sometimes, it could be recovered by applying kicks to the beam (using the strip-line shaker normally used for beam excitation in betatron tune measurements or using the injection kicker). The shift of the beam orbit does not affect the events.

Study of the effect turning the localized pumps (LP) and distributed ion pumps (DIPs) on and off had shown that with all LP turned off no sudden lifetime loss had happened although some systematic lifetime reduction was observed due to lower vacuum. It was concluded that DIPs are the main source of the dust.

Although the dust events had been known for many years, the phenomenon eluded explanation. The basic processes defining dust trapping, the lifetime of the dust particles, and the dust-beam interaction were described by Zimmermann [1] and in [2]. For the PEP-II B-factory the model [2] predicts that most of the dust particles should

\footnotetext{
*Permanent address: IHEP, Beijing, China.

${ }^{\dagger}$ Permanent address: LBL, Berkeley, CA, USA.
}

burn down in $50 \mu \mathrm{s}$, by many orders of magnitude faster than in reality, and only microscopic silica needles may have chance to survive longer.

We have found such an explanation of the long time dust events possible but exceptional. One can expect that a needle with sufficiently large positive charge $Q$ (see discussion below) in the field of the electron beam would be electrostatically aligned along the beam line. The balance of ionization heating and radiation cooling in this case would be the same as for a spherical dust particle.

We found that the large amplitude oscillations can be the cause of the dust long lifetime. In the next section we discuss formulas and equations used later for calculations. The formulas describing the main processes are, basically, the same as in the previous analysis [1] but refined in several aspects. Results of simulations of the one dimensional (1D) vertical motion of the dust particles in the field of a flat beam are discussed in the third section. The lifetime for large amplitude of oscillations is, as we expected, substantially longer than it was obtained before [2]. However, especially for 1A PEP-II beam current, the dust lifetime is still too short and the simulations were modified in Sec. IV to describe the 2D dust motion. With such modification, we were able to get the time of the dust event comparable with that in observations. Effect on the beam lifetime is discussed in Sec. V where we corrected the estimate of the beam lifetime used in [1].

TABLE I. BEPC parameters.

\begin{tabular}{lcc}
\hline \hline & PEP-II & BEPC \\
\hline Beam energy, $E_{B}, \mathrm{GeV}$ & 9.1 & 2.2 \\
Frequency rf, $f_{\mathrm{rf}}, \mathrm{MHz}$, & 500 & 200.0 \\
Beam current, $I_{B}, \mathrm{~mA}$ & 1000 & 65 \\
Bunch population $N_{b}$, & $2 \times 10^{10}$ & $0.81 \times 10^{10}$ \\
RF voltage/cavity, $V_{\mathrm{rf}}, \mathrm{MV}$, & 0.8 & 0.8 \\
Circumference $2 \pi R, \mathrm{~m}$ & 2200 & 240 \\
Bunch spacing $s_{b}, \mathrm{~m}$ & 1.2 & 6.0 \\
$\mathrm{~V} / \mathrm{H}$ tune,$\nu_{y / x}$ & $22.6 / 23.6$ & $4.75 / 8.72$ \\
$\mathrm{rms} \sigma_{x / y}, \mu \mathrm{m}$ & $800 / 150$ & $497 / 28$ \\
$\mathrm{rms} \sigma_{z}, \mathrm{~cm}$ & 1.0 & 3.0 \\
Beam pipe radius, $b, \mathrm{~cm}$ & 3.5 & 4.0 \\
\hline \hline
\end{tabular}


Elsewhere numerical examples are given for the Ti dust particles.

\section{MAIN PROCESSES}

In most cases, the velocity of the dust is relatively low and the dust collides with several bunches while crossing the beam line. Even at 1 A PEP-II current, the typical time of crossing the beam is by an order of magnitude larger than the time duration of the ion gap in the bunch train. For BEPC parameters, that is true even in a single-bunch operation. That allows us to consider coasting uniformly charged beam with the linear density $N_{b} / s_{b}$.

A dust particles is ejected, probably, from the ion pumps. It may have initial charge or acquires some charge by the photo effect due to direct or scattered SR. The charge of the dust particle approaching the beam grows rapidly due to ionization by the electrons in the tail of the beam distribution. The process starts at several transverse rms $\sigma_{\perp}$ and, as a result, the dust particle is trapped in the field of the electron beam. The motion of the dust particle, its radius $a$, charge $Q$, and temperature $T$ has to be defined selfconsistently by the beam ionization losses, radiative cooling, and field emission of positive ions. In this section we summarize formulas used below in our calculations.

\section{A. Dust dynamics}

The dust particle moves in the potential $U(x, y)$ of the Gaussian coasting beam with rms $\sigma_{x}$ and $\sigma_{y}$. The potential in the dimensionless coordinates $x, y$ in units of $\sigma_{x}$ and $\sigma_{y}$, respectively, can be written as

$$
\begin{aligned}
U(x, y)= & -\frac{N_{b} Q e^{2}}{s_{b}} \int_{0}^{\infty} \frac{d \lambda}{\lambda \sqrt{1+2 \lambda \sigma_{x}^{2}} \sqrt{1+2 \lambda \sigma_{y}^{2}}} \\
& \times \exp \left[-\frac{\lambda \sigma_{x}^{2} x^{2}}{1+2 \lambda \sigma_{x}^{2}}-\frac{\lambda \sigma_{y}^{2} y^{2}}{1+2 \lambda \sigma_{y}^{2}}\right] .
\end{aligned}
$$

Potential $U(x, y)$ is known as Bassetti-Erskin potential and can be expressed in terms of the error function of complex argument [3]. For small amplitude, the motion is oscillations with the frequency $\Omega_{0}$ proportional to the beam current. For the BEPC averaged beam current $I_{B}, \Omega_{0}$ is low compared with the revolution frequency $f_{\text {rev }}$. That is true for BEPC parameters for any filling pattern, even for a single bunch in the ring. Therefore, the filling pattern does not affect the dust events as it was found in the experiments.

\section{B. Evaporation}

The lifetime of the dust particle is limited by evaporation of neutral atoms and their thermal dissociation. Evaporation of ions may be important as well at large temperature. The number of nuclei $A$ in the spherical dust particle is defined by the radius $a$,

$$
A=\frac{4 \pi a^{3}}{3} \frac{\rho}{m_{N}} A_{a} .
$$

Here $m_{N}$ is mass of a nucleon, $A_{a}$ is atomic weight, and $\rho$ is the dust density ( $a^{3} \rho / A_{a}$ is dimensionless). The rate of evaporation is proportional [4] to the vapor pressure $P_{v}(T)$,

$$
\frac{d A}{d t}=-\frac{4 \pi c a^{2}}{\sqrt{2 \pi k_{B} T M_{a} c^{2}}} P(T) .
$$

$A_{a}$ is atomic number, $M_{a}$ is the mass of an atom, $c$ and $k_{B}$ are the velocity of light and the Boltzmann constant, respectively, and we neglect reflectivity (for most metals it is very small). In our simulations we use $P(T)$ interpolating experimental data on the temperature dependence of pressure. Pressure in Pascal for Ti is [5]

$$
P(T)=1986.71 T^{2.2898} e^{-\left(\epsilon_{v} / k B T\right)}, \quad \text { for } \mathrm{Ti},
$$

where the enthalpy of evaporation per atom $\epsilon_{v}=$ $4.412 \mathrm{eV}$. Equation (4) gives better parametrization of the experimental data than parametrization quoted in [1]. Comparison is shown in Fig. 1.

The total pressure $P_{\text {tot }}=P(T)+\Delta P$ for a dust particle has to be corrected by the additional term due to the surface tension,

$$
\Delta P=\frac{2 \gamma_{s}}{a} \frac{\rho_{v}}{\rho},
$$

where the last factor is the ratio of the vapor density $\rho_{v}$ to the density $\rho$ of the dust particle.

The lifetime of large particles due to evaporation can be quite large at a moderate temperature. For $1 \mu$ Ti particle, $A \simeq 10^{13}$ and $d A / d t \simeq 3.4 \times 10^{8}$ at $T=1500 \mathrm{~K}$ but grows rapidly to $d A / d t \simeq 1.2 \times 10^{12}$ at the melting point $T_{M}=$ $1941 \mathrm{~K}$.

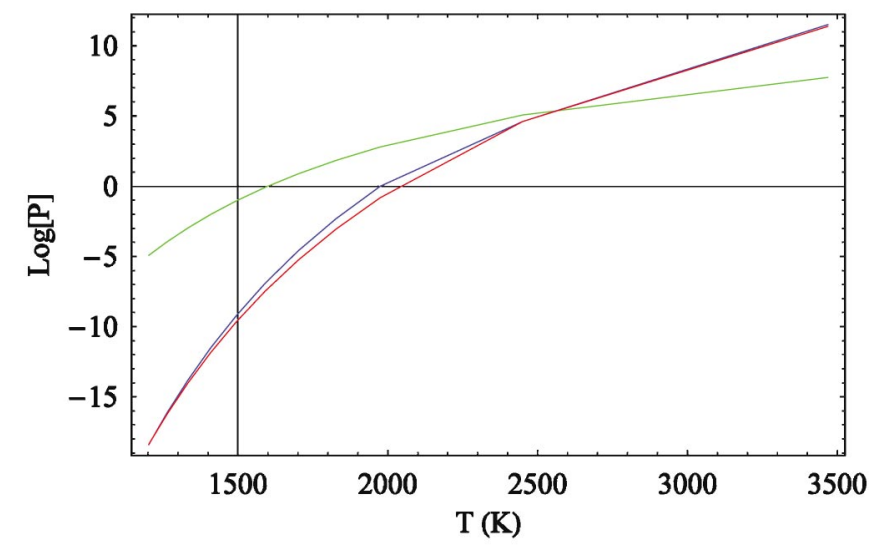

FIG. 1. (Color) The pressure $P(T)$ in Pascal for Ti. Interpolating function, see Eq. (4), is shown by the blue and the experimental data by the black lines. The red line is pressure parametrization according to Ref. [1]. 
However, the actual lifetime of dust particles may be defined by stability to fission. Stability to fission, and the dynamics of the dust particle depend on its charge $Q /, e$ and the temperature $T$.

\section{Heating}

For a particle with the transverse offset $x, y$ (in units of transverse rms $\sigma_{x}$ and $\sigma_{y}$, respectively), the heating is due to deposited energy

$$
\Delta E_{i}=\Delta E_{T} N_{b}\left(\frac{\pi a^{2}}{2 \pi \sigma_{x} \sigma_{y}}\right) e^{-1 / 2\left(x^{2}+y^{2}\right)},
$$

where $\Delta E_{T}$ is ionization energy loss per single electron of the ultrarelativistic beam colliding with the dust particle [6],

$$
\Delta E_{T}=\Delta E_{Q} \ln \left[2\left(\frac{m_{e} c^{2}}{\hbar \omega_{p}}\right)^{2}\left(\frac{Q r_{e}}{a}+\frac{1}{2}\left(\frac{\hbar \omega_{p}}{m c^{2}}\right)^{2}\right)\right] .
$$

The plasmon energy [6]

$$
\hbar \omega_{p}=28.816 \sqrt{\frac{Z_{a}}{A_{a}} \rho},
$$

and

$$
\Delta E_{Q}=2 \pi r_{e}^{2} m c^{2} N_{A v} \frac{4 a}{3} \frac{Z_{a}}{A_{a}} \rho .
$$

Here $r_{e}$ and $m c^{2}$ are the classical radius and the rest energy of an electron, $\rho$ is the density in $\mathrm{g} / \mathrm{cm}^{3}$, and $N_{A v}=$ $6.02 \times 10^{23}$ is the Avogadro constant. Other parameters are explained in Tables I and II.

The cutoff energy in Eq. (7) is taken equal to the potential of the dust particle $Q e^{2} / a$. Another limitation on the cutoff energy is set by comparison of $l(\varepsilon)$, the free path of the secondary electrons with the energy $\varepsilon$, with the dust size $a$. The experimental energy dependence can be fitted by

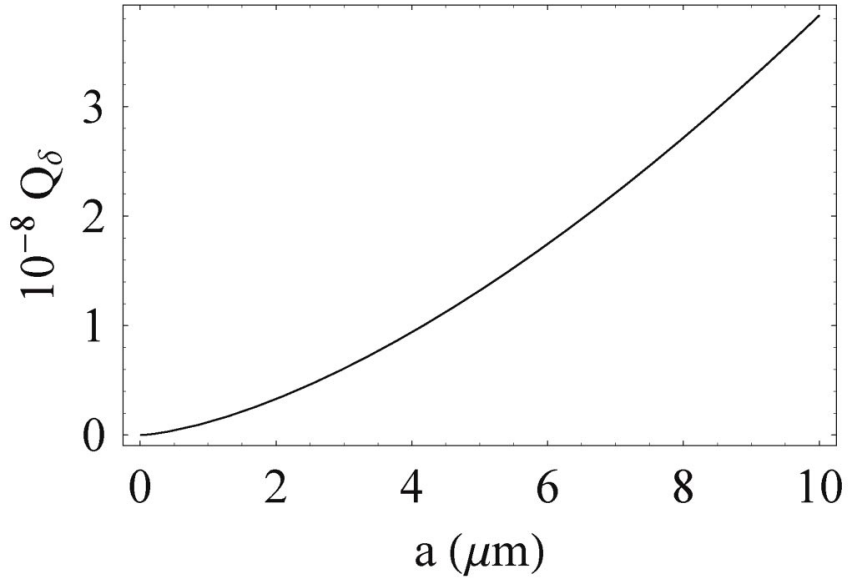

FIG. 2. Parameter $Q_{\delta}(a)$ given by the limit on the free path length of secondary electrons, see text.

$$
l(\varepsilon)=\frac{0.412}{\rho} \varepsilon^{n(\varepsilon)}, \quad n(\varepsilon)=1.265-0.0954 \ln (\varepsilon),
$$

where $l$ is in $\mathrm{cm}, \varepsilon$ is in $\mathrm{MeV}$, and $\rho$ in $\mathrm{g} / \mathrm{cm}^{3}$. The constrain on the free path length of the secondary electron $l(\varepsilon)<a$ can be written in the form $\varepsilon<Q_{\delta} e^{2} / a$. The quantity $Q_{\delta}$ defined in this way depends on the radius $a$. Then, $Q$ in Eq. (7) has to be replaced by $Q_{\text {eff }}=$ $\max \left\{Q, Q_{\delta}\right\}$ where $Q_{\delta}(a)$ for $\mathrm{Ti}$ found numerically is shown in Fig. 2. More careful consideration has to define $l(\varepsilon)$ as the distance to the surface of the dust particle from the point $\left(x_{s}, y_{s}\right)$ where the secondary electron was generated averaged over $\left(x_{s}, y_{s}\right)$. This correction is, however, small.

The heating due to ionization loss competes with cooling by the thermal radiation,

$$
\left(\frac{d E}{d t}\right)_{\mathrm{rad}}=4 \pi a^{2} \sigma_{S B} T^{4} \Phi(\mu)
$$

TABLE II. Some relevant parameters.

\begin{tabular}{lc}
\hline \hline Elements & $\{\mathrm{Al}, \mathrm{Cu}, \mathrm{Ti}, \mathrm{Si}\}$ \\
\hline Atomic weight, $A_{a}$ & $\{27,64,48,28.1\}$ \\
Atomic number, $Z_{a}$ & $\{13,29,22,14\}$ \\
Density, $\rho, g / \mathrm{cm}^{3}$ & $\{2.7,8.96,4.54,2.5\}$ \\
Melting point, $T_{M}, \mathrm{~K}$ & $\{933,1352,1941,1983\}$ \\
Heat capacity, $C_{T}, \mathrm{~J} /(\mathrm{mol} \mathrm{K})$ & $\{29.3,35.6,33.7,72.47\}$ \\
Surface tension, $\gamma_{S}, \mathrm{mN} / \mathrm{m}$ & $\{840,1150,1427,10000\}$ \\
Ionization potential $I_{P}, \mathrm{eV}$ & $\{5.98,7.72,6.82,8.15\}$ \\
Enthalpy of fission $\epsilon_{f}, \mathrm{~kJ} / \mathrm{mol}$ & $\{10.7,13.1,15.45,50.2\}$ \\
Enthalpy of vaporization $\epsilon_{v}, \mathrm{~kJ} / \mathrm{mol}$ & $\{293,300,425,359\}$ \\
Conversion & $\mathrm{eV}=1.6 \times 10^{-19} \mathrm{~J}$ \\
Coefficients & $\mathrm{eV} / \mathrm{cm}^{2}=1.6 \times 10^{-12} \mathrm{mN} / \mathrm{m}$ \\
& $\mathrm{Torr}=0.83 \times 10^{15} \mathrm{eV} / \mathrm{cm}^{3}$ \\
\hline \hline
\end{tabular}


where $\left.\sigma_{S B}=3.53 \times 10^{7} \mathrm{eV}(\mathrm{sec} \mathrm{cm})^{2}\right)^{-1} \mathrm{~K}^{-4}$ is the constant of the black body radiation. The form factor $\Phi(\mu)$ takes into account suppression of the long wave length radiation with $\omega<\omega_{c}$, where $\omega_{c} a / c \simeq 1$. The suppression in the Mie theory is approximately described by the factor $[1,7]$

$$
\Phi(\mu)=\frac{1}{1+C_{w} /(a T)},
$$

where $a$ is in $\mathrm{cm}$ and $T$ in $K$.

Additional cooling is provided by the evaporation of neutral atoms,

$$
\left(\frac{d E}{d t}\right)_{n}=-\epsilon_{v} \frac{d A}{d t}
$$

The temperature variation per collision with a bunch is described by the following equation:

$$
\frac{d T}{d t}=\left[\frac{c}{s_{b}} \Delta E_{i}-\left(\frac{d E}{d t}\right)_{\mathrm{rad}}-\left(\frac{d E}{d t}\right)_{n}\right]\left(\frac{3 A_{a}}{4 \pi a^{3} C_{T} \rho}\right),
$$

where $C_{T}$ is the heat capacity per mole.

\section{Charge variation}

Equation (7) describes only the part of the ionization losses which go to the dust heating. Another part, with the energy loss larger than $Q_{\text {eff }} e^{2} / a+\left(\hbar \omega_{p}\right)^{2} / 2 m c^{2}$, is taken away by the secondary electrons which escape the dust particle. That increases the charge $Q$ of the dust particle with the rate

$$
\begin{aligned}
\left(\frac{d Q}{d t}\right)_{i}= & \left(\frac{c}{s_{b}}\right) \Delta E_{Q}\left(\frac{N_{B}}{Q_{\mathrm{eff}} e^{2} / a+(1 / 2)\left(\hbar \omega_{p} / m c^{2}\right)^{2}}\right) \\
& \times\left(\frac{\pi a^{2}}{2 \pi \sigma_{x} \sigma_{y}}\right) e^{-(1 / 2)\left(x^{2}+y^{2}\right)} .
\end{aligned}
$$

The thermal dissociation of evaporated neutral atoms at the surface of the charged dust particle can stop further ionization. Electrons are captured by the positively charged dust reducing $Q$. The rate of the process is given by the Richardson's formula [8]

$\left(\frac{d Q}{d t}\right)_{d}=\frac{2\left(2 \pi a k_{B} T\right)^{2} M_{i}}{(2 \pi \hbar)^{3}} e^{-1 / k_{B} T\left[I-W+\epsilon_{v}-\left(e^{2} / a\right) \sqrt{Q}\right]+\left(\Delta C_{p} / k_{B}\right)}$.

The Schottky term $\propto \sqrt{Q}$ decreases the potential barrier due to electric field of the charged particle. Here $I$ is the ionization potential, $W$ is the work function for Ti (and affinity for $\mathrm{Si}$ ), $M_{i}$ is the ion mass, and

$$
\Delta C_{P}=\int_{0}^{T} \frac{d \tau}{\tau^{2}} \int_{0}^{\tau} C_{P}\left(T^{\prime}\right) d T^{\prime}
$$

Interpolating experimental data for the heat capacity $C_{P}$, we get

$$
\Delta C_{P}=\frac{A_{\text {int }}}{N_{\mathrm{Av}}} \frac{\ln \left[1+B_{\mathrm{int}} T\right]}{B_{\mathrm{int}}}\left[1+\frac{1}{B_{\mathrm{int}} T}\right] \frac{e V}{K},
$$

where parameters $A_{\text {int }}=0.2265 \mathrm{~J} / \mathrm{K}, B_{\text {int }}=0.006081 / \mathrm{K}$ were found for Ti by fitting.

The total charge variation is given by Eqs. (15) and (16):

$$
\frac{d Q_{i}}{d t}=\left(\frac{d Q}{d t}\right)_{i}-\left(\frac{d Q}{d t}\right)_{d}
$$

The field of the beam can cause polarization of the dust particles. The effect, however, is small and can be neglected.

\section{E. Fission}

The dust particle melts when temperature exceeds the melting point $T>T_{M}$. Then the deposited energy goes to the latent heat of transition from solid to liquid state

$$
\frac{d E_{M}}{d t}=\left[\frac{c}{s_{b}} \Delta E_{i}-\left(\frac{d E}{d t}\right)_{\mathrm{rad}}-\left(\frac{d E}{d t}\right)_{n}\right]
$$

converting the dust particle to a droplet. After melting, the droplet can split into two smaller droplets. The process of fission has the threshold charge $Q_{\text {th }}$ defined by the equilibrium of the electrostatic potential energy and the potential energy of the surface tension [9]. For small deformations, the threshold $Q_{\mathrm{th}}$ is given by

$$
\frac{Q_{\mathrm{th}}^{2} r_{e} m c^{2}}{16 \pi \gamma_{S} a^{3}}=1 \text {. }
$$

(The threshold is lower for the large deformation.)

The energy released in fission of a dust particle with initial radius $a$ and charge $Q>Q_{\text {th }}$ depends on the size and the charge $x /, Q$ and $(1-x) /, Q$ of the droplets in the final state. The gain is maximum for fission in halves, $x=1 / 2$, see the upper plot in Fig. 3. The bottom plot shows dependence of the threshold $Q$ to fission in halves as function of the radius $a$ of the dust particle.

The typical energy released in fission $E_{f}$ is large and, because the beam potential at large distances is only logarithmic, the final droplets acquiring large momentum can hit the walls of the beam pipe. Therefore, in the dust events with long lifetime, the dust particle either has to stay below the melting point or, if it melts, should not go to fission. Figure 4 shows the ratio of the released energy to the depth of the potential, well. The product of fission survive provided the ratio is less than one.

Other effects such as diffusion due to multiple collisions and capture of the photo electrons may affect the dust dynamics. Estimates show that these effects are small for moderate $Q$ and we do not include them in simulations.

\section{1D DYNAMICS}

Equations of motion in the potential (1), and Eqs. (3), (14), (19), and (20), give the system of differential equa- 

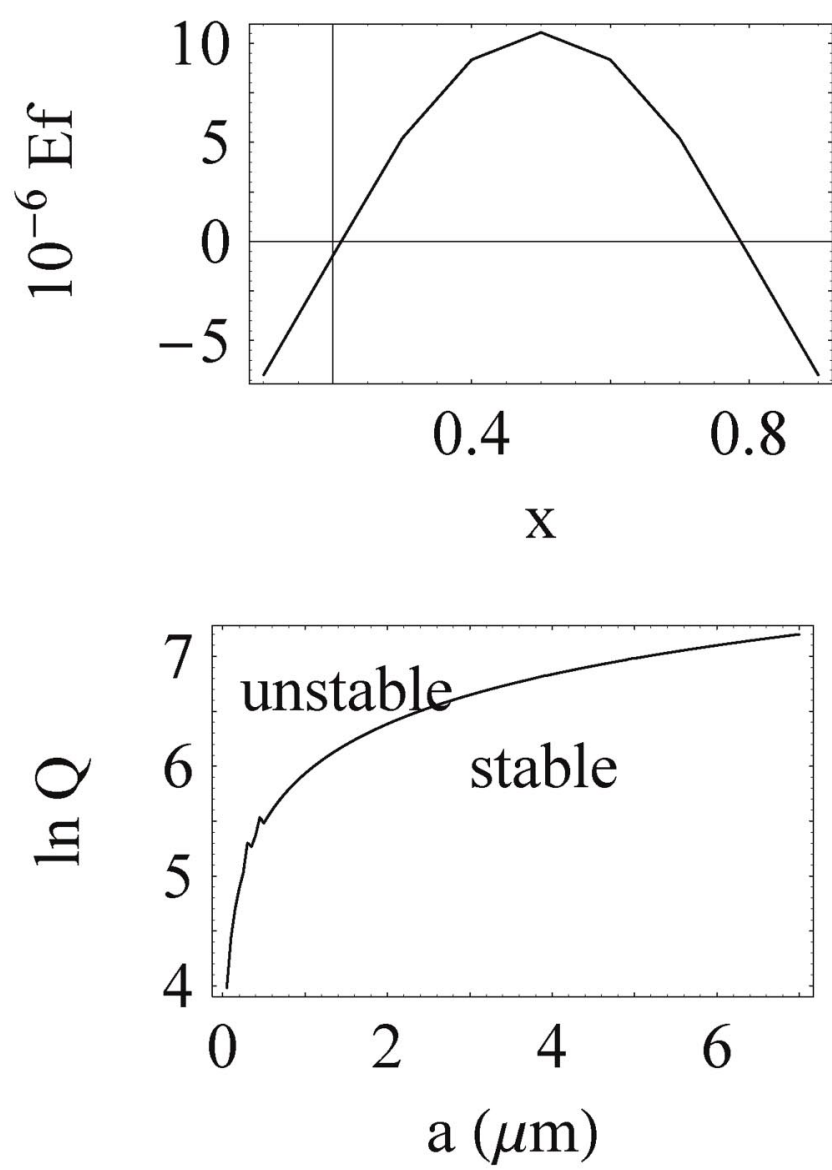

FIG. 3. Upper plot: energy released in fission to particles with the masses equal to the fraction $x$ and $1-x$ of the initial mass calculated with $Q=10^{6}$ and the initial dust radius $a=1 \mu \mathrm{m}$. Bottom plot: the threshold charge $Q$ as function of the radius $a$ of the dust particle.

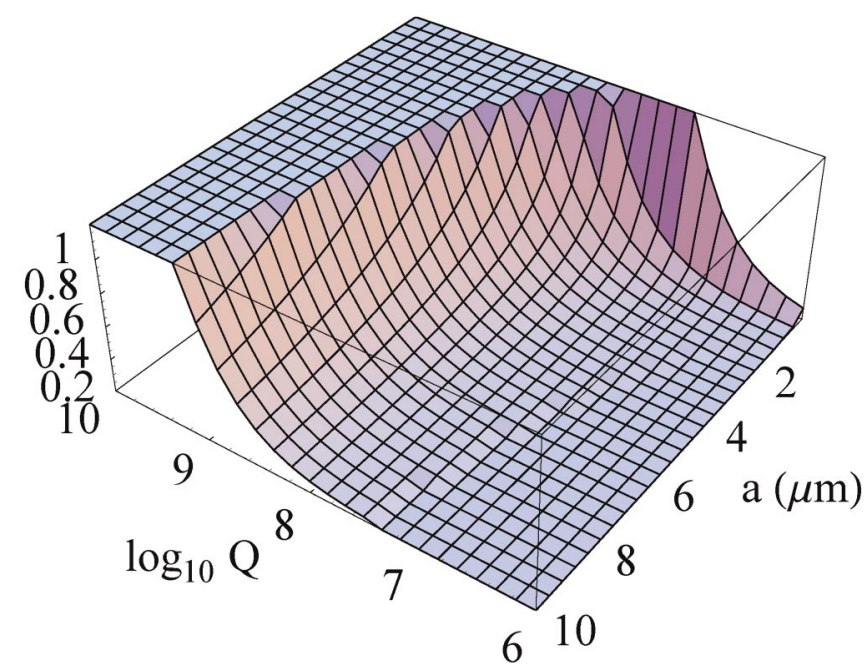

FIG. 4. (Color) The ratio of the energy released in the fission to the depth of the potential well as function of the charge $Q$ and the beam pipe radius $a$. The product of decay does not hit the beam pipe provided the depicted parameter is less than one. tions for coordinates $x, y$, dust radius $a$, charge $Q$, and temperature $T$.

For a flat beam, it seems reasonable to consider only 1D vertical oscillations of the dust particle.

Dynamics of the dust particle is quite complicated. The particle with low $Q$ approaching the beam increases $Q$ and $T$ in the first encounter with the beam. After the first crossing of the beam, the charge $Q$ and velocity are large. While away from the beam, the ionization heating by the beam is negligible and continuing radiation and evaporation provides cooling of the dust particle. The particle returns to the beam with reduced $T, Q$, and velocity. Eventually, after several crossings, the temperature increases to a value larger than the melting point $T_{M}$ and some energy is stored as latent heat of transition. After the crossing, the temperature remains equal $T_{M}$ until the latent energy goes to zero due to radiation and evaporation. Because of high rate of dissociation of neutrals at high temperature, $Q$ may drop to a lower value. The returning force decreases, and the amplitude of oscillations of the particle increases. That may, generally speaking, lead to stable oscillations with the lifetime limited by evaporation. However, even if the fission does not take place, the particle lifetime cannot be long. The rate of evaporation $d A / d t$ is high. For example, for $1 \mu$ Ti dust particle, $A=$ $2 \times 10^{12}$, and evaporation rate $d A / d t=1.5 \times 10^{11} 1 / \mathrm{s}$ at $T=2000 \mathrm{~K}$. Therefore, one could expect the lifetime of only few seconds for $a=1 \mu$.

The typical case is illustrated in Fig. 5 for $a=0.2 \mu$ and BEPC parameters. Depicted results are obtained using MATHEMATICA by solving the system of equations described above. The charge $Q$ and temperature $T$ slowly rise after each crossing and are reduced when particle is away from the beam. Growing $Q$ leads to adiabatic decrease of the amplitude of oscillations enhancing further growth of $Q$ and $T$. When $Q$ exceeds the fission threshold, the temperature already $T>T_{M}$, and fission takes place producing such a large kick to the products of decay that they hit the wall. The lifetime is only $30 \mathrm{~ms}$.

The same happens even faster for larger particles and at larger PEP-II 1 A current. The fission repeats several times. The large number of small particles could reduce the beam lifetime. However, their own lifetime due to evaporation is too short. The ions, the final product of the process, are unstable in the train with the sufficiently long ion gap and disappear in few revolution periods.

\section{2D DYNAMICS}

At large currents such as the PEP-II 1 A beam current, the dust crossing the beam within 1-2 $\sigma_{y}$ is heated to temperature larger than the melting point $T_{M}$ and, after melting, decays to smaller fragments which evaporate shortly. That is consistent with the conclusion in Ref. [1] but contradicts to the observations. Thus, it is essential to consider the 2D motion. 

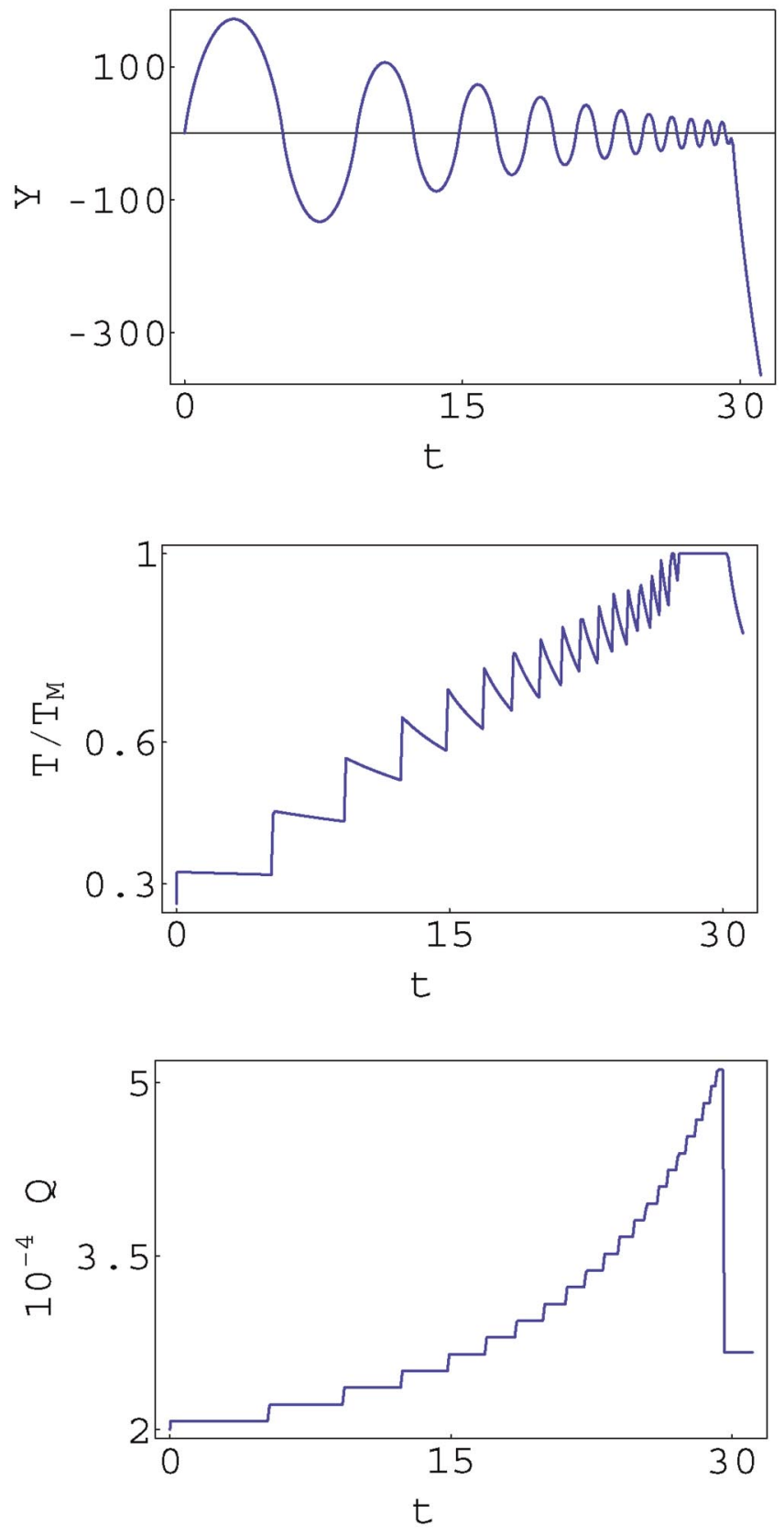

FIG. 5. (Color) 1D dynamics of $a=0.2 \mu \mathrm{m}$ Ti dust particle for BEPC parameters. The lifetime of the dust particle is limited by fission due to the growing charge $Q$ : when $T$ exceeds the melting point $T_{M}$, the dust particle decays and the products hit the wall. The vertical amplitude $Y$ is in units of $\sigma_{y}$ and time is in ms.

It is instructive to consider the axially symmetric case where the azimuthal component of the angular momentum $L_{\phi}=p_{\phi} r$ is constant while the charge $Q$ may vary. The radial potential of the particle has the minimum at the radius $r_{m}$,

$$
\frac{1}{r_{m}^{2}}=\frac{Z_{0} e I_{B} Q M_{d}}{2 \pi L_{\phi}^{2}}
$$

where $I_{B}$ is the average beam current and $Z_{0}=120 \pi \Omega$. Initial $p_{\phi}(0) \simeq p(0)$ and $L_{\phi} \simeq p(0) b$ are defined by the initial momentum $p(0)$ and the beam pipe radius $b$. The dust in the minimum of the potential is trapped at the distance $r \simeq r_{m}$ from the beam. The distance $r_{m}<\sigma_{\perp}$ if the charge

$$
Q>\left[\frac{Z_{0} e I_{b} \sigma_{\perp}}{4 \pi b^{2}}\right]^{-1} E_{0} .
$$

Numerical calculations show that the typical $Q$ is lower than that and the trapped particle does not approach the beam closer than several $\sigma_{\perp}$.

For a flat beam, the axial component $L_{\phi}$ is not an integral of motion. The trajectory of the dust particle in this case is a rotating ellipse and is confined between two circles. As in the axially symmetric case, the particle can stay away from the beam interacting only with the tails of the beam distribution. That means that the heating of the particle is reduced and it can live longer but the effect on the beam lifetime is also reduced. The trade-off of these two factors defines the range of parameters where the dust events can take place.

Result of 2D simulations are shown in Figs. 6 and 7 for BEPC, and Fig. 8 for PEP-II. Interpolation of the slow growth of temperature in the last case shows that the particle would survive another $10 \mathrm{~s}$. We checked accuracy of simulations calculating the azimuthal $L_{\phi}$ for a round beam where we put $\sigma_{y}=\sigma_{x}$. $L_{\phi}$ is preserved during the $2000 \mathrm{~ms}$ of tracking with computer accuracy.

\section{BEAM LIFETIME}

The beam lifetime is defined either by the energy loss $\Delta E / E_{B}$ of the beam electrons exceeding the energy acceptance of the ring $\delta_{\max }$ or by the large angle scattering. The
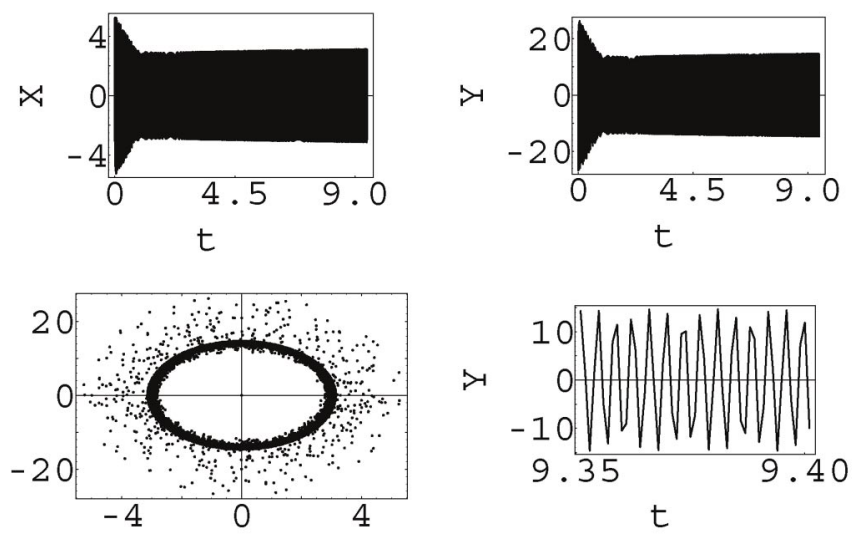

FIG. 6. Trajectories $x(t)$ and $y(t)$ of $5 \mu \mathrm{m}$ Ti particle for BEPC parameters vs time in seconds. Initial conditions $x(0)=3.0$, $p_{x}(0)=0, y(0)=0.0, v_{y}(0)=6.56 \mathrm{~m} / \mathrm{s}, T=1500 \mathrm{~K}, Q_{0}=$ $10^{8}$. The bottom row shows the trajectory in the $(x, y)$ plane and the zoomed part of the trajectory for the last $50 \mathrm{~ms}$ of tracking. 

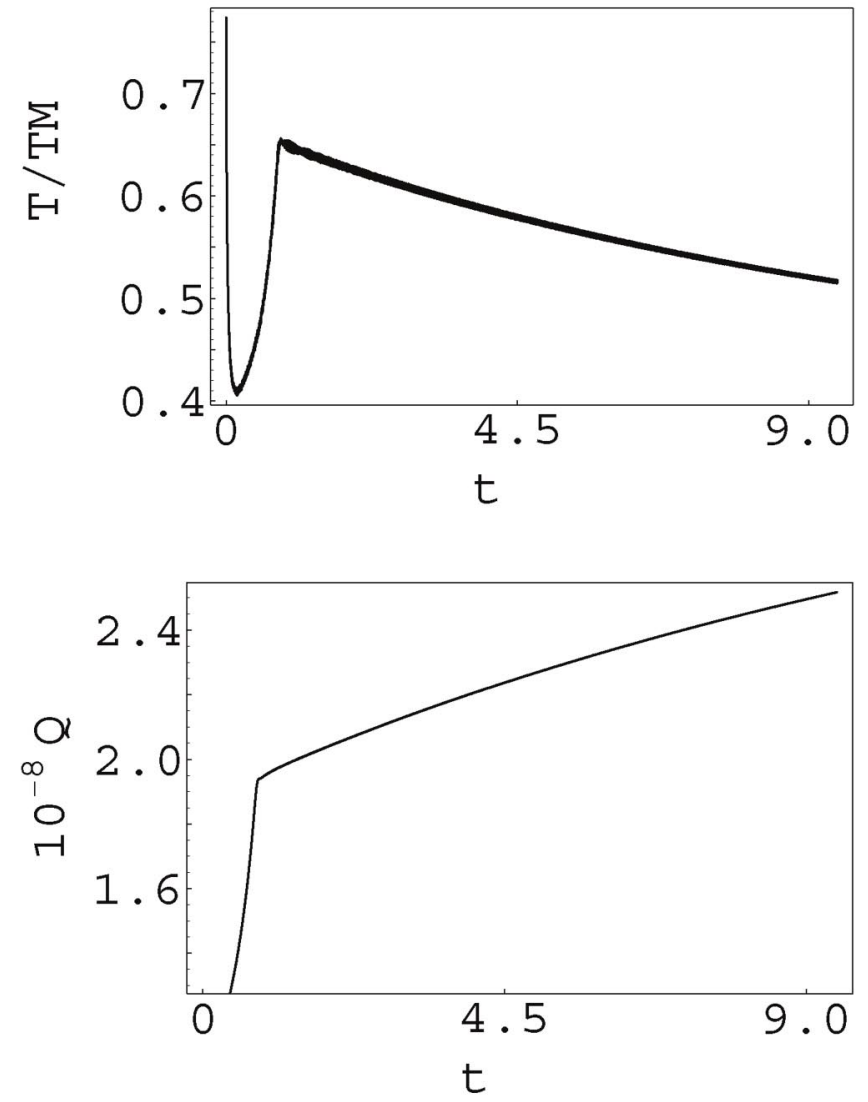

FIG. 7. Variation of the temperature (in units of $T_{M}$ ) and the charge $Q$ with time in seconds for the same case as in Fig. 6 . estimate shows that, for the typical $\delta_{\max } \simeq 10^{-2}$ the first constrain is stronger.

The energy loss can be due to bremsstrahlung with the impact parameter $b_{0}>a$ (the process named duststrahlung [1]) or due to bremsstrahlung on the nuclei of the dust particle with $b_{0}<a$.

The lifetime due to duststrahlung for the 2D large amplitude oscillations is exponentially long, see the appendix. The dominant process defining the beam lifetime in this case is the bremsstrahlung on nuclei of the dust particle. The cross section of this process [10] is

$$
d \sigma(\omega)=4 Z_{a}^{2} \alpha_{0} r_{e}^{2} \frac{d \omega}{\omega} \frac{E^{\prime}}{E}\left(\frac{E}{E^{\prime}}+\frac{E^{\prime}}{E}-\frac{2}{3}\right)\left[\ln \frac{2 E E^{\prime}}{m \omega}-\frac{1}{2}\right],
$$

where $E^{\prime}=E-\hbar \omega$. The lifetime

$$
\frac{1}{\tau_{B}}=\int_{\omega_{m}}^{\infty} d \sigma(\omega) f_{\mathrm{rev}} n \pi a^{2} \frac{4 a}{3}\langle d f\rangle,
$$

where $\langle d f\rangle$ is the distribution functions of the beam particles crossing the dust, and $n=\left(\rho / A_{a}\right) N_{\mathrm{Av}}$ is the number of nuclei per unit volume. For the dust particle moving periodically over ellipse with axes $A_{e}$ and $B_{e}$ (in units $\sigma_{x}$ and $\sigma_{y}$, respectively), $\langle d f\rangle$ should be averaged over the period:

$$
\begin{aligned}
\langle d f\rangle & =\int \frac{d \phi}{2 \pi} \frac{1}{2 \pi \sigma_{x} \sigma_{y}} e^{-(1 / 2)\left(A_{e}^{2} \cos ^{2} \phi+B_{e}^{2} \sin ^{2} \phi\right)} \\
& =\frac{1}{2 \pi \sigma_{x} \sigma_{y}} e^{-(1 / 4)\left(A_{e}^{2}+B_{e}^{2}\right)} I_{0}\left[\frac{1}{4}\left(A_{e}^{2}-B_{e}^{2}\right)\right],
\end{aligned}
$$
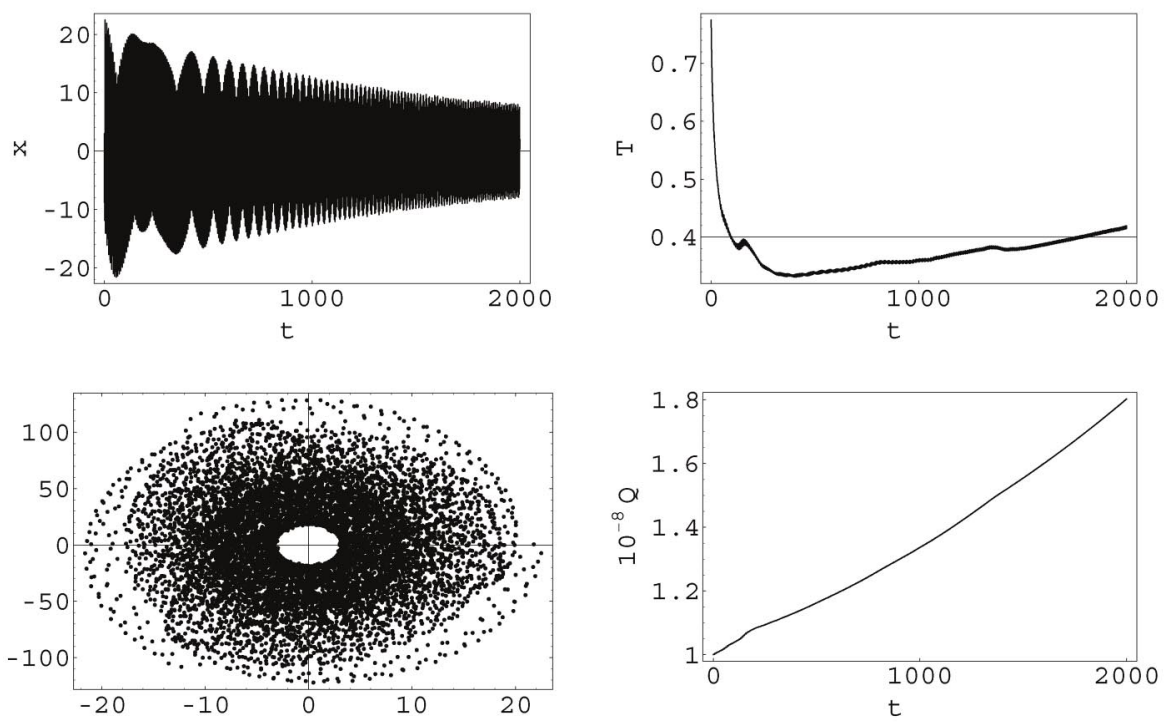

FIG. 8. Tracking of $5 \mu$ Ti particle for PEP-II parameters. Initial conditions are: $x(0)=3.0, v_{y}(0)=41.5 \mathrm{~m} / \mathrm{s}, y(0)=v_{x}(0)=0$, $T(0)=1500 \mathrm{~K}, Q(0)=10^{8}$. Depicted are $x$ and $y$ in units of $\sigma_{x}=835 \mu \mathrm{m}$ and $\sigma_{y}=145 \mu \mathrm{m}$, respectively, $Q$ in units of $10^{8}, T(t)$ in units of the melting point $T_{M}=1941 \mathrm{~K}$, time in ms. Radius $a$ does not change with accuracy $10^{-12}$ during 2000 ms. Interpolation of $T(t)$ predicts that $T_{M}$ will be reached in another $10 \mathrm{~s}$. However, that is uncertain because $Q$ has not reached saturation yet. Beating is due to mismatch of initial conditions. 
where $I_{0}$ is the Bessel function of imaginary argument. If $B_{e} \gg A_{e}$ (see, for example, Figs. 6 and 8),

$$
\langle d f\rangle=\frac{1}{2 \pi \sigma_{x} \sigma_{y}} e^{-\left(A_{e}^{2} / 2\right)} \sqrt{\frac{2}{\pi B_{e}^{2}}}
$$

That means that the electrons are lost when the dust particle crosses the horizontal plane where it is relatively close to the beam. Equation (25) gives the estimate

$$
\begin{aligned}
\frac{1}{\tau_{B}}= & \frac{16}{3} \alpha_{0}\left(Z_{a} r_{e}\right)^{2} f_{\text {rev }} \ln \left(\frac{1}{\delta_{m}}\right)\left[\ln \left(\frac{2 \gamma \lambda_{c}}{Z_{a} r_{e} \delta_{m}}\right)-\frac{1}{2}\right] \\
& \times \frac{4 a n}{3} \frac{\pi a^{2}}{2 \pi \sigma_{x} \sigma_{y}} e^{-A_{e}^{2} / 2} \sqrt{\frac{2}{\pi\left(B_{e}^{2}-A_{e}^{2}\right)}} .
\end{aligned}
$$

As an example let us take $a=5 \mu$ Ti dust particle, acceptance $\delta_{m}=0.01$, and $A_{e}=4.0, B_{e}=20$. Then, for PEP-II parameters $(E=9 \mathrm{GeV}, 2 \pi R=2.2 \mathrm{~km})$, Eq. (28) gives $1 / \tau_{B}=0.79 \times 10^{-6} \mathrm{~s}^{-1}$, consistent with the experimental $1 / \tau_{B}=1.4 \times 10^{-6}$ corresponding to $10 \%$ drop of the nominal lifetime $(20 \mathrm{~h})$. For BEPC and $5 \mu$ Ti particle, Eq. (28) gives $1 / \tau_{B}=0.06 \times 10^{-3} 1 / \mathrm{s}$ with $A_{e}=4.0$, $B_{e}=15$, and $1 / \tau_{B}=0.4 \times 10^{-3} 1 / \mathrm{s}$ with $A_{e}=3.5$, $B_{e}=15$, what has to be compared with $1 / \tau_{B}=0.13 \times$ $10^{-3}$ in observations.

\section{CONCLUSION}

The dust events were observed during the last 30 years in many machines. Although main relevant processes are well understood [1] the present models predict the lifetime of dust particles by many order of magnitude shorter than in the experiments. In the special case of long silica needles the lifetime can be longer. In this case, it is defined by the time to build up charge sufficient to align to needle along the beam. In our study we have found that the lifetime of a dust particles trapped and oscillating with amplitudes less or comparable to the beam transverse rms is, indeed, very short [2]. The lifetime of such particle is limited both by fission and evaporation. Typically, the melting point is reached and the fission takes place much faster then dust evaporates. After fission, the fragments of decay obtain large momentum and hit the beam pipe wall or go to fission again. Eventually, fission and evaporation produce ions. The estimates show that even heavy ions do not survive long in the bunch trains with a sufficiently large ion gap. Although the islands of stability do exist for any bunch train, they occupy a small fraction of the ring, especially for rings with small revolution period. The lifetime of ions in the islands of stability is also limited by the secondary ionization [1] and by the space charge of accumulated ions [11].

The situation may be different for the dust oscillating with large amplitudes. Such particles spend most of the time outside of the beam and may have enough time to be cooled by radiation. The simulations show that, with BEPC parameters, such particles can have a lifetime of the order of $100 \mathrm{~ms}$, long but still much shorter than in observations. Our attempts to prolong the dust lifetime by varying within the reasonable limits the input parameters (ionization potential, enthalpy, chemical composition, and the initial size of the particles, etc.) were unsuccessful. We came to the conclusion that it is essential to consider 2D motion where particles can remain at large distances from the beam all the time. The lifetime of a dust particle in this case can be large. However, the interaction of such dust particles with the beam is reduced. We show that the effect of the duststrahlung in this case is small and the beam lifetime is defined by the bremsstrahlung on the dust nuclei. The beam lifetime in our examples is in reasonable agreement with the experiment. Although we were unable to explore the whole range of parameters (beam currents, geometry, initial charge and velocity of the dust particles, the size and composition of the particle, etc.) limiting the dust events, we give realistic examples where both lifetime of the dust and the beam lifetime are comparable with observations.

\section{ACKNOWLEDGMENTS}

This work was supported by Department of Energy Contract No. DE-AC02-76SF00515 and National Foundation of Natural Sciences Contract No. 10275079A050501.

\section{APPENDIX: LIFETIME DUE TO DUSTSTRAHLUNG}

The lifetime was estimated in Ref. [1] using formulas for beamstrahlung borrowed from the theory of beam-beam collisions. We found that for moderate $a<10 \mu$ the estimate has to be modified.

Effect on the lifetime in the ultrarelativistic case [12] depends on the parameter $Q r_{e} / b_{0}$ equal to the ratio of the deflection angle $\alpha \simeq Q r_{e} /\left(\gamma b_{0}\right)$ to the radiation angle $\theta_{r} \simeq$ $1 / \gamma$ where $\gamma$ is a relativistic factor. The spectrum of radiation for $Q r_{e} / b_{0} \gg 1$ is described by the formulas for the synchrotron radiation and was assumed in the previous analysis [1]. The simulations show that more realistic is another limit $Q r_{e} / b_{0} \ll 1$ where the spectrum of radiated photons is [12]

$$
\begin{aligned}
\frac{d E_{\omega}}{d \omega}= & \frac{e^{2} \omega}{2 \pi c^{3}} \int_{\omega /\left(2 \gamma^{2}\right)} \frac{d \omega^{\prime}}{\left(\omega^{\prime}\right)^{2}}\left|w\left(\omega^{\prime}\right)\right|^{2}\left[1-\frac{\omega}{\omega^{\prime} \gamma^{2}}\right. \\
& \left.+\frac{1}{2}\left(\frac{\omega}{\omega^{\prime} \gamma^{2}}\right)^{2}\right] .
\end{aligned}
$$

Here $w(\omega)$ is the Fourier transform of the transverse acceleration $w(t)$,

$$
w(t)=\frac{Q e^{2}}{m \gamma} \frac{b_{0}}{\left(b_{0}^{2}+c^{2} t^{2}\right)^{3 / 2}} .
$$


Explicitly, $w(\omega)$ is given in terms of the modified Bessel function $K_{1}$,

$$
w(\omega)=\frac{2 Q r_{e} c}{\gamma b_{0}} \frac{\omega b_{0}}{c} K_{1}\left(\frac{\omega b_{0}}{c}\right) .
$$

The average energy loss is small. The loss of particles in this case is due to rare but strong fluctuations where a photon is radiated with the frequency $\omega>\omega_{\min }$, $\hbar \omega_{\min } / E_{B}=\delta_{\max }$.

Equation (A1) defines the number of photons $d N_{\gamma}\left(b_{0}\right)=d \omega d E_{\omega} /(\hbar \omega)$. The lifetime $\tau\left(b_{0}\right)$ of a particle with coordinates $x, y$ is

$$
\frac{1}{\tau\left(b_{0}\right)}=f_{\text {rev }} \int_{\omega_{\min }}^{\infty} d N_{\gamma}\left(b_{0}\right)
$$

where $b_{0}=\sqrt{\left(x-x_{0}\right)^{2}+\left(y-y_{0}\right)^{2}}$ and $x_{0}, y_{0}$ are coordinates of the dust center.

Equation (A4) can be written in the form

$$
\frac{1}{\tau\left(b_{0}\right)}=\frac{\alpha_{0} f_{0}}{\pi}\left(\frac{2 Q r_{e}}{b_{0}}\right)^{2} x_{m}^{2} \int_{1}^{\infty} d z K_{1}^{2}\left(x_{m} z\right)\left[\frac{2 z}{3}-1+\frac{1}{z}-\frac{2}{3 z^{2}}\right] \text {, }
$$

where $x_{m}=\delta_{m} b_{0} /\left(2 \gamma \lambda_{c}\right), \alpha_{0}=1 / 137$, and $\lambda_{c}=r_{e} / \alpha_{0}$ is the Compton wave length.

The integral is exponentially small,

$$
\frac{1}{\tau\left(b_{0}\right)} \simeq \frac{\alpha_{0} f_{0}}{\pi}\left(\frac{2 Q r_{e}}{b_{0}}\right)^{2} \frac{\pi}{8 x_{m}} e^{-2 x_{m}}, \quad x_{m} \gg 1
$$

for large $x_{m}$. For small $x_{m}$, it can be estimated as

$$
\frac{1}{\tau\left(b_{0}\right)} \simeq \frac{\alpha_{0} f_{0}}{\pi}\left(\frac{2 Q r_{e}}{b_{0}}\right)^{2} \frac{2}{3} \ln \left(1 / x_{m}\right), \quad x_{m} \ll 1 .
$$

The beam life $\tau_{B}$ can be obtained by averaging Eq. (A5) over Gaussian distribution $d f(x, y)$ of electrons of the beam. The accurate estimate of the beam lifetime due to duststrahlung can be done introducing additional integration

$$
\begin{aligned}
\frac{1}{\tau_{B}}= & \int_{a}^{\infty} 2 b_{0} d b_{0} \delta\left[\left(x-x_{0}\right)^{2}+\left(y-y_{0}\right)^{2}-b_{0}^{2}\right] \\
& \times \int d f[x, y] \frac{1}{\tau(x, y)}
\end{aligned}
$$

and carrying out integration over $d x d y$ first.

$$
\begin{aligned}
& \text { If } r_{0}=\sqrt{x_{0}^{2}+y_{0}^{2}} \text { is of the order of } \sigma_{\perp}, \\
& \qquad \frac{1}{\tau_{B}} \simeq \frac{2 \alpha_{0} f_{0}}{3 \pi}\left(\frac{2 Q r_{e}}{\sigma_{y}}\right)^{2} \ln \left(\frac{2 \gamma \lambda_{c}}{\delta_{m} a}\right) .
\end{aligned}
$$

Dependence on $a$ in this case is only logarithmic.

The lifetime given by Eq. (A9) is comparable with that for PEP-II parameters at $Q \simeq 10^{8}$. However, the lifetime of the dust particle with such $Q$ in the core of the beam is only few $\mu$ s. The beam life due to duststrahlung for stable particles is too long to be noticeable in experiments.

In the opposite case of large amplitudes of oscillations $a_{0} \gg \sigma_{\perp}$ only few particles in the tails of the distribution encounter the dust particle,

$$
\frac{1}{\tau_{B}} \simeq \frac{\alpha_{0} f_{0}}{4}\left(\frac{Q r_{e}}{\sigma_{y}}\right)^{2}\left(\frac{a^{2}}{\sigma_{\perp} a_{0}}\right) e^{-\delta_{m} a_{0} / \gamma \lambda_{c}} e^{-a_{0}^{2} / 2 \sigma_{\perp}^{2}} .
$$

The lifetime due to duststrahlung in the last case is exponentially long.

[1] F. Zimmermann, PEP-II AP Note 8-94, 1994.

[2] F. Zimmermann, J. Seeman, and M. Zolotorev, Report No. SLAC-PUB-6788, 1995.

[3] M. Bassetti and G. Erskin, Report No. CERN ISR TH/8006, 1989.

[4] L.D. Landau and E. M. Lifshitz, Statistical Physics (Pergamon Press, Oxford, U.K., New York, 1969).

[5] G. Kaye and T. Laby, Tables of Physical and Chemical Constants (Longman, New York, 1995), 16th ed.

[6] Special issue on Review of Particle Physics [Eur. Phys. J. C 15, 1 (2000)].

[7] H. Van de Hulst, Light Scattering by Small Particles (Wiley and Sons, Inc., New York, 1957).

[8] E. U. Condon and H. Odishaw, Handbook of Physics (McGraw-Hill Book Company, New York, 1967), 2nd ed.

[9] L. Landau and E. Lifshitz, Electrodynamics of Continuous Media (Pergamon Press, Oxford, U.K., New York, 1984).

[10] V. Berestetskii, E. Lifshitz, and L. Pitaevskii, Quantum Electrodynamics (Pergamon Press, Oxford, U.K., New York, 1982).

[11] D. Villevald and S. Heifets, SLAC PEP-II AP Note 18-93, 1993.

[12] L. D. Landau and E. M. Lifshitz, The Classical Theory of Fields (Pergamon Press, Oxford, U.K., New York, 1975), 4th ed. 